\title{
Dome-Shaped Head
}

National Cancer Institute

\section{Source}

National Cancer Institute. Dome-Shaped Head. NCI Thesaurus. Code C124527.

A head having the shape of a dome. 